\title{
ORDER-BOUNDED OPERATORS FROM VECTOR-VALUED FUNCTION SPACES TO BANACH SPACES
}

\author{
MARIAN NOWAK \\ Faculty of Mathematics, Computer Science and Econometrics \\ University of Zielona Góra \\ Szafrana 4A, 65-516 Zielona Góra, Poland \\ E-mail: M.Nowak@wmie.uz.zgora.pl
}

\begin{abstract}
Let $E$ be an ideal of $L^{0}$ over a $\sigma$-finite measure space $(\Omega, \Sigma, \mu)$. For a real Banach space $\left(X,\|\cdot\|_{X}\right)$ let $E(X)$ be a subspace of the space $L^{0}(X)$ of $\mu$-equivalence classes of strongly $\Sigma$-measurable functions $f: \Omega \rightarrow X$ and consisting of all those $f \in L^{0}(X)$ for which the scalar function $\|f(\cdot)\|_{X}$ belongs to $E$. Let $E(X)^{\sim}$ stand for the order dual of $E(X)$. For $u \in E^{+}$let $D_{u}\left(=\left\{f \in E(X):\|f(\cdot)\|_{X} \leq u\right\}\right)$ stand for the order interval in $E(X)$. For a real Banach space $\left(Y,\|\cdot\|_{Y}\right)$ a linear operator $T: E(X) \rightarrow Y$ is said to be order-bounded whenever for each $u \in E^{+}$the set $T\left(D_{u}\right)$ is norm-bounded in $Y$. In this paper we examine order-bounded operators $T: E(X) \rightarrow Y$. We show that $T$ is order-bounded iff $T$ is $\left(\tau\left(E(X), E(X)^{\sim}\right),\|\cdot\|_{Y}\right)$-continuous. We obtain that every weak Dunford-Pettis operator $T: E(X) \rightarrow Y$ is order-bounded. In particular, we obtain that if a Banach space $Y$ has the Dunford-Pettis property, then $T$ is order-bounded iff it is a weak Dunford-Pettis operator.
\end{abstract}

1. Introduction and preliminaries. P. G. Dodds $[\mathrm{D}]$ introduced and examined orderbounded operators from a vector lattice $E$ to a Banach space $Y$. Recall that a linear operator $T: E \rightarrow Y$ is called order-bounded if the set $T([-u, u])$ is norm-bounded in $Y$ for every $0 \leq u \in E$. M. Duboux [Du] extended some of Dodd's results to the setting of $Y$ being a locally convex space. Next, Z. Ercan [E] obtained some properties of orderbounded operators from a vector lattice $E$ to a topological vector space $Y$.

In this paper we consider order-bounded operators from a vector-valued function space $E(X)$ to a Banach space $Y$.

For the terminology concerning vector lattices and function spaces we refer to $\left[\mathrm{AB}_{1}\right]$, $\left[\mathrm{AB}_{3}\right]$ and $[\mathrm{KA}]$. Given a topological vector space $(L, \tau)$ by $(L, \tau)^{*}$ we will denote its

2000 Mathematics Subject Classification: 47B38, 47B07, 46E40, 46A20.

Key words and phrases: vector-valued function spaces, Köthe-Bochner spaces, order-bounded operators, order intervals, the Dunford-Pettis property, weak Dunford-Pettis operators.

The paper is in final form and no version of it will be published elsewhere. 
topological dual. We denote by $\sigma(L, K)$ and $\tau(L, K)$ the weak topology and the Mackey topology for a dual system $(L, K)$ resp. By $\mathbb{N}$ and $\mathbb{R}$ we will denote the sets of all natural and real numbers.

Throughout the paper we assume that $(\Omega, \Sigma, \mu)$ is a complete $\sigma$-finite measure space and $L^{0}$ denotes the corresponding space of $\mu$-equivalence classes of all $\Sigma$-measurable real valued functions. Let $\chi_{A}$ stand for the characteristic function of a set $A$. Let $E$ be an ideal in $L^{0}$ with supp $E=\Omega$ and let $E^{\sim}$ stand for the order dual of $E$.

Let $\left(X,\|\cdot\|_{X}\right)$ be a real Banach space and let $S_{X}$ stand for the unit sphere of $X$. By $L^{0}(X)$ we denote the set of $\mu$-equivalence classes of all strongly $\Sigma$-measurable functions $f: \Omega \rightarrow X$. For $f \in L^{0}(X)$ let us set $\tilde{f}(\omega):=\|f(\omega)\|_{X}$ for $\omega \in \Omega$. Let

$$
E(X)=\left\{f \in L^{0}(X): \widetilde{f} \in E\right\} .
$$

A subset $H$ of $E(X)$ is said to be solid whenever $\widetilde{f}_{1} \leq \widetilde{f}_{2}$ and $f_{1} \in E(X), f_{2} \in H$ imply $f_{1} \in H$. A linear topology $\tau$ on $E(X)$ is said to be locally solid if it has a local base at zero consisting of solid sets. A linear topology $\tau$ on $E(X)$ that is at the same time locally solid and locally convex will be called a locally convex-solid topology on $E(X)$ (see $\left.\left[\mathrm{N}_{1}\right]\right)$.

Recall that the algebraic tensor product $E \otimes X$ is the subspace of $E(X)$ spanned by the functions of the form $u \otimes x,(u \otimes x)(\omega)=u(\omega) x$, where $u \in E, x \in X$.

For each $u \in E^{+}$the set $D_{u}=\{f \in E(X) \widetilde{f} \leq u\}$ will be called an order interval in $E(X)($ see $[\mathrm{BL}])$.

Following $[\mathrm{D}]$ we are now ready to define some class of linear operators.

Definition. Let $E$ be an ideal of $L^{0}$ and $\left(X,\|\cdot\|_{X}\right)$ and $\left(Y,\|\cdot\|_{Y}\right)$ real Banach spaces. A linear operator $T: E(X) \rightarrow Y$ is said to be order-bounded whenever for each $u \in E^{+}$ the set $T\left(D_{u}\right)$ is norm-bounded in $Y$.

Now we recall terminology concerning the duality theory of the function spaces $E(X)$ as set out in $[\mathrm{B}],[\mathrm{BL}],\left[\mathrm{N}_{1}\right],\left[\mathrm{N}_{2}\right]$.

For a linear functional $F$ on $E(X)$ let us put

$$
|F|(f)=\sup \{|F(h)|: h \in E(X), \widetilde{h} \leq \widetilde{f}\} \quad \text { for } f \in E(X) .
$$

The set

$$
E(X)^{\sim}=\left\{F \in E(X)^{\#}:|F|(f)<\infty \text { for all } f \in E(X)\right\}
$$

will be called the order dual of $E(X)$ (here $E(X)^{\#}$ denotes the algebraic dual of $E(X)$ ).

For $F_{1}, F_{2} \in E(X)^{\sim}$ we will write $\left|F_{1}\right| \leq\left|F_{2}\right|$ whenever $\left|F_{1}\right|(f) \leq\left|F_{2}\right|(f)$ for all $f \in E(X)$. A subset $A$ of $E(X)^{\sim}$ is said to be solid whenever $\left|F_{1}\right| \leq\left|F_{2}\right|$ with $F_{1} \in E(X)^{\sim}$ and $F_{2} \in A$ imply $F_{1} \in A$.

Now we consider absolute weak topologies on $E(X)$ and $E(X)^{\sim}$. For each $F \in E(X)^{\sim}$ let

$$
\rho_{F}(f)=|F|(f) \quad \text { for } f \in E(X) .
$$

We define the absolute weak topology $\sigma \mid\left(E(X), E(X)^{\sim}\right)$ on $E(X)$ as the locally convex topology generated by the family $\left\{\rho_{F}: F \in E(X)^{\sim}\right\}$ of seminorms. Then $|\sigma|\left(E(X), E(X)^{\sim}\right)$ is the topology of uniform convergence on sets of the form $\left\{G \in E(X)^{\sim}:|G| \leq|F|\right\}$ for every $F \in E(X)^{\sim} \quad\left(\right.$ see $\left[\mathrm{N}_{1}\right.$, Section 4$\left.]\right)$. 
For each $f \in E(X)$ let

$$
\rho_{f}(F)=|F|(f) \quad \text { for } F \in E(X)^{\sim} .
$$

We define the absolute weak topology $|\sigma|\left(E(X)^{\sim}, E(X)\right)$ on $E(X)^{\sim}$ as the locally convex topology generated by the family $\left\{\rho_{f}: f \in E(X)\right\}$ of seminorms. Clearly $|\sigma|(E(X) \sim, E(X))$ is the topology of uniform convergence on the family of all order intervals $D_{u}$, where $u \in E^{+}$.

In particular, if $\left(E,\|\cdot\|_{E}\right)$ is a Banach function space then the space $E(X)$ provided with the norm $\|f\|_{E(X)}:=\|\widetilde{f}\|_{E}$ is a Banach space and it is usually called the KötheBochner space. It is well known that $\left(E(X),\|\cdot\|_{E(X)}\right)^{*}=E(X) \sim($ see $[\mathrm{BL}, \S 3$, Lemma 12]).

2. Characterization of order-bounded operators. It is known that on every vector lattice $E$ one can define the so-called order-bounded topology $\tau_{0}$ as the finest locally convex topology on $E$ for which every order interval is a bounded set (see [Na]). A local base at zero for $\tau_{0}$ is given by the class of all absolutely convex subsets of $E$ that absorb all order bounded sets in $E$. It is known that $\tau_{0}$ coincides with the Mackey topology $\tau\left(E, E^{\sim}\right)($ see $[\mathrm{F}, 811(\mathrm{c})])$.

Now let $\mathcal{B}_{0}$ be the family of all absolutely convex subsets of $E(X)$ that absorb every order interval in $E(X)$. Then $\mathcal{B}_{0}$ is a local base at zero for a locally convex topology $\tau_{0}$ on $E(X)$, which will be called an order-bounded topology on $E(X)$.

We are ready to characterize order-bounded operators $T: E(X) \rightarrow Y$ in terms of the $\left(\tau_{0},\|\cdot\|_{Y}\right)$-continuity of $T$.

Theorem 2.1. For a linear operator $T: E(X) \rightarrow Y$ the following statements are equivalent:

(i) $T$ is order-bounded.

(ii) $T$ is $\left(\tau_{0},\|\cdot\|_{Y}\right)$-continuous.

Proof. (i) $\Rightarrow$ (ii). Assume that $T$ is order bounded and let $r>0$ be given. We shall show that there is $V \in \mathcal{B}_{0}$ such that $T(V) \subset B_{Y}(r)\left(=\left\{y \in Y:\|y\|_{Y} \leq r\right\}\right)$. In fact, let $V=T^{-1}\left(B_{Y}(r)\right)=\left\{f \in E(X):\|T(f)\|_{Y} \leq r\right\}$. Since $T(V)=T\left(T^{-1}\left(B_{Y}(r)\right)\right) \subset B_{Y}(r)$, it is enough to show that $V$ absorbs every order interval in $E(X)$. Indeed, for given $u \in E^{+}$there is $r_{u}>0$ such that $T\left(D_{u}\right) \subset B_{Y}\left(r_{u}\right)$. Taking $\lambda_{u}=\frac{r}{r_{u}}$ for $f \in D_{u}$ we get $\left\|T\left(\lambda_{u} f\right)\right\|_{Y} \leq r$, so $\lambda_{n} f \in V$. This means that $\lambda_{u} D_{u} \subset V$.

(ii) $\Rightarrow$ (i). Assume that $T$ is $\left(\tau_{0},\|\cdot\|_{Y}\right)$-continuous. Then there is $V \in \mathcal{B}_{0}$ such that $T(V) \subset B_{Y}(1)$. Moreover, given $u \in E^{+}$there is $\lambda_{u}>0$ such that $\lambda_{u} D_{u} \subset V$. Hence $T\left(\lambda_{u} D_{u}\right) \subset B_{Y}(1)$, so $T\left(D_{u}\right) \subset B_{Y}\left(1 / \lambda_{u}\right)$, as desired.

As an application of Theorem 2.1 we obtain:

Corollary 2.2. The order-bounded topology $\tau_{0}$ on $E(X)$ coincides with the Mackey topology $\tau\left(E(X), E(X)^{\sim}\right)$, i.e., $\tau_{0}=\tau\left(E(X), E(X)^{\sim}\right)$.

Proof. In view of Theorem 2.1, $\left(E(X), \tau_{0}\right)^{*}=E(X)^{\sim}$, so by the Mackey-Arens theorem $\tau_{0} \subset \tau\left(E(X), E(X)^{\sim}\right)$.

To show that $\tau\left(E(X), E(X)^{\sim}\right) \subset \tau_{0}$, let $W \in \mathcal{B}_{\tau(E(X), E(X) \sim)}$ (= the local base at zero for $\left.\tau\left(E(X), E(X)^{\sim}\right)\right)$. It is enough to show that $W$ absorbs every order interval in $E(X)$. 
Since the Mackey topology $\tau\left(E(X), E(X)^{\sim}\right)$ is locally solid (see [ $\mathrm{N}_{2}$, Theorem 3.7]), $W={ }^{0} A(=\{f \in E(X):|F(f)| \leq 1$ for all $F \in A\})$, where $A$ is an absolutely convex, solid and $\sigma\left(E(X)^{\sim}, E(X)\right)$-compact subset of $E(X)^{\sim}$. But in view of [ $\mathrm{N}_{2}$, Theorem 3.5] $A$ is $|\sigma|(E(X) \sim, E(X))$-bounded, so for each $f \in E(X)$ we have:

$$
\sup \{|F|(f): F \in A\}<\infty \text {. }
$$

Observe that

$$
{ }^{0} A=\{f \in E(X):|F|(f) \leq 1 \text { for all } F \in A\} .
$$

In fact, let $f \in{ }^{0} A$, i.e., $|F(f)| \leq 1$ for all $F \in A$. It is enough to show that $|F|(f) \leq 1$ for all $F \in A$. Given $F_{0} \in A$, for each $G \in E(X)^{\sim}$ with $|G| \leq\left|F_{0}\right|$ we have that $G \in A$ because $A$ is a solid subset of $E(X)^{\sim}$. Hence $|G(f)| \leq 1$. But by $\left[\mathrm{N}_{1}\right.$, Lemma 2.1] $F_{0} \mid(f)=\sup \left\{|G(f)|: G \in E(X)^{\sim},|G| \leq\left|F_{0}\right|\right\}$, so we obtain that $\left|F_{0}\right|(f) \leq 1$, as desired.

Now, we are ready to show that $W$ absorbs every order interval in $E(X)$. In fact, let $u \in E^{+}$and $x_{0} \in S_{X}$. Hence in view of $(*) \sup \left\{|F|\left(u \otimes x_{0}\right): F \in A\right\}=\lambda_{u}<\infty$. Then for $f \in D_{u}$ and all $F \in A$ we have:

$$
|F|\left(\frac{1}{\lambda_{u}} f\right)=\frac{1}{\lambda_{u}}|F|(f) \leq \frac{1}{\lambda_{u}}|F|\left(u \otimes x_{0}\right) \leq 1,
$$

so $\frac{1}{\lambda_{u}} f \in W$, i.e., $D_{u} \subset \lambda_{u} W$.

We say that a sequence $\left(f_{n}\right)$ in $E(X)$ is uniformly convergent to $f \in E(X)$ (in symbols, $\left.f_{n} \rightarrow f(r u)\right)$, if there exist $r \in E^{+}$and a sequence $\left(\varepsilon_{n}\right)$ of positive numbers with $\varepsilon_{n} \downarrow 0$ such that $\left\|f_{n}(\omega)-f(\omega)\right\|_{X} \leq \varepsilon_{n} r(\omega) \mu$-a.e. on $\Omega$.

Making use of Theorem 2.1 and Corollary 2.2 we get:

ThEOREM 2.3. For a linear operator $T: E(X) \rightarrow Y$ the following statements are equivalent:

(i) $T$ is order bounded.

(ii) $T$ is $\left(\tau\left(E(X), E(X)^{\sim}\right),\|\cdot\|_{Y}\right)$-continuous.

(iii) $T$ is $\left(\sigma\left(E(X), E(X)^{\sim}\right), \sigma\left(Y, Y^{*}\right)\right)$-continuous.

(iv) If $\widetilde{f}_{n} \leq u$ for some $u \in E^{+}$and all $n \in \mathbb{N}$ and $f_{n} \rightarrow 0$ for $|\sigma|\left(E(X), E(X)^{\sim}\right)$, then $\sup _{n}\left\|T\left(f_{n}\right)\right\|_{Y}<\infty$.

(v) If $f_{n} \rightarrow 0(r u)$, then $\sup _{n}\left\|T\left(f_{n}\right)\right\|_{Y}<\infty$.

In particular, for a Banach function space $\left(E,\|\cdot\|_{E}\right)$ the statements $(\mathrm{i})-(\mathrm{v})$ are equivalent to the following:

(vi) $T$ is $\left(\|\cdot\|_{E(X)},\|\cdot\|_{Y}\right)$-continuous.

Proof. (i) $\Leftrightarrow$ (ii). It follows from Theorem 2.1 and Corollary 2.2.

(ii) $\Leftrightarrow($ iii). See [W, Corollary 11.1.3, Corollary 11.2.6].

(i) $\Rightarrow$ (iv). It is obvious.

(iv) $\Rightarrow$ (i). Assume that (iv) holds and (i) fails. Hence there is $u_{0} \in E^{+}$such that $\sup \left\{\|T(f)\|_{Y}: f \in E(X), \tilde{f} \leq u_{0}\right\}=\infty$. It follows that one can find a sequence $\left(f_{n}\right)$ in $E(X)$ such that $f_{n} \leq u_{0}$ for all $n \in \mathbb{N}$ and $\left\|T\left(f_{n}\right)\right\|_{Y} \geq n^{2}$ for all $n \in \mathbb{N}$. Hence putting $h_{n}=\frac{1}{n} f_{n}$ for $n \in \mathbb{N}$, we have $\widetilde{h}_{n} \leq \frac{1}{n} u_{0} \leq u_{0}$ and $\left\|T\left(h_{n}\right)\right\|_{Y} \geq n$ for $n \in \mathbb{N}$. We shall 
show that $h_{n} \rightarrow 0$ for $|\sigma|\left(E(X), E(X)^{\sim}\right)$. Indeed, let $F \in E(X)^{\sim}$, and $x_{0} \in S_{X}$. Then

$$
\rho_{F}\left(h_{n}\right)=|F|\left(h_{n}\right) \leq|F|\left(\frac{1}{n}\left(u_{0} \otimes x_{0}\right)\right)=\frac{1}{n}|F|\left(u_{0} \otimes x_{0}\right) \rightarrow 0 .
$$

Hence $h_{n} \rightarrow 0$ for $\sigma \mid\left(E(X), E(X)^{\sim}\right)$, so by (iv) $\sup _{n}\left\|T\left(h_{n}\right)\right\|_{Y}<\infty$. This contradiction establishes that (iv) $\Rightarrow$ (i).

$(\mathrm{i}) \Rightarrow(\mathrm{v})$. Assume that (i) holds and (v) fails. Then there exists a sequence $\left(f_{n}\right)$ in $E(X)$ such that $f_{n} \rightarrow 0(r u)$ and $\sup _{n}\left\|T\left(f_{n}\right)\right\|_{Y}=\infty$. This means that there exist $r \in E^{+}$and a sequence $\left(\varepsilon_{n}\right)$ with $\varepsilon_{n} \downarrow 0$ such that $\tilde{f}_{n} \leq \varepsilon_{n} r \leq \varepsilon_{n} r$ for all $n \in \mathbb{N}$. Hence by (i) $\sup _{n}\left\|T\left(f_{n}\right)\right\|_{Y}<\infty$, and we get a contradiction.

$(\mathrm{v}) \Rightarrow(\mathrm{i})$. Assume that (i) fails. Hence there exists $u_{0} \in E^{+}$such that

$$
\sup \left\{\|T(f)\|_{Y}: f \in E(X), \tilde{f} \leq u_{0}\right\}=\infty .
$$

So, there exists a sequence $\left(f_{n}\right)$ in $E(X)$ such that $\tilde{f}_{n} \leq u_{0}$ and $\left\|T\left(f_{n}\right)\right\|_{Y} \geq n^{2}$ for all $n \in \mathbb{N}$. Denoting $h_{n}=\frac{1}{n} f_{n}$ for $n \in \mathbb{N}$ we get $\widetilde{h}_{n} \leq \frac{1}{n} u_{0}$ for $n \in \mathbb{N}$, i.e., $h_{n} \rightarrow 0(r u)$ and $\left\|T\left(h_{n}\right)\right\|_{Y} \geq n$ for all $n \in \mathbb{N}$. It follows that (v) does not hold.

Now assume that $\left(E,\|\cdot\|_{E}\right)$ is a Banach function space. Then $\left(E(X),\|\cdot\|_{E(X)}\right)^{*}=$ $E(X)^{\sim}$ and the Mackey topology $\tau\left(E(X), E(X)^{\sim}\right)$ coincides with the $\|\cdot\|_{E(X)}$-norm topology. Hence (ii) $\Leftrightarrow($ vi).

Recall that a Banach space $Y$ is said to have the Dunford-Pettis property if for sequences $\left(y_{n}\right)$ in $Y$ and $\left(y_{n}^{*}\right)$ in $Y^{*}, y_{n}^{*}\left(y_{n}\right) \rightarrow 0$ whenever $y_{n} \stackrel{(w)}{\longrightarrow} 0$ in $Y$ and $y_{n}^{*} \stackrel{(w)}{\longrightarrow} 0$ in $Y^{*}$ (see $\left[\mathrm{AB}_{1}\right.$, Section 19]).

Following ( $\left[\mathrm{AB}_{2}\right],\left[\mathrm{AB}_{3}\right.$, Section 19]) we say that a linear operator $T: E(X) \rightarrow Y$ is a weak Dunford-Pettis operator if $f_{n} \rightarrow 0$ in $E(X)$ for $\sigma\left(E(X), E(X)^{\sim}\right)$ and $y_{n}^{*} \stackrel{(w)}{\longrightarrow} 0$ in $Y^{*}$ imply $y_{n}^{*}\left(T\left(f_{n}\right)\right) \rightarrow 0$.

As an application of Theorem 2.3 we get (see [E, Theorem 1.4]):

\section{TheOREM 2.4.}

(i) Every weak Dunford-Pettis operator $T: E(X) \rightarrow Y$ is order-bounded.

(ii) If $Y$ has the Dunford-Pettis property, then every order-bounded operator $T: E(X)$ $\rightarrow Y$ is a weak Dunford-Pettis operator.

Proof. (i) Assume that $T: E(X) \rightarrow Y$ is a weak Dunford-Pettis operator and it is not order-bounded. Hence by Theorem 2.3 there exists a sequence $\left(f_{n}\right)$ in $E(X)$ such that $f_{n} \rightarrow 0(r u)$ and $\sup _{n}\left\|T\left(f_{n}\right)\right\|_{Y}=\infty$. This means that there exist $u_{0} \in E^{+}$and a sequence $\left(\varepsilon_{n}\right)$ with $\varepsilon_{n} \downarrow 0$ such that $\widetilde{f}_{n} \leq \varepsilon_{n} u_{0}$ for all $n \in \mathbb{N}$ and the set $\left\{T\left(f_{n}\right): n \in \mathbb{N}\right\}$ in $Y$ is not weakly bounded. Hence there exist $y_{0}^{*} \in Y^{*}$ and a subsequence $\left(f_{k_{n}}\right)$ of $\left(f_{n}\right)$ such that

$$
\left|y_{0}^{*}\left(T\left(f_{k_{n}}\right)\right)\right|>n \quad \text { for all } n \in \mathbb{N} \text {. }
$$

One can observe that $f_{n} \rightarrow 0$ for $\sigma\left(E(X), E(X)^{\sim}\right)$. Indeed, let $F \in E(X)^{\sim}$ and $x_{0} \in S_{X}$. Then

$$
\left|F\left(f_{n}\right)\right| \leq|F|\left(f_{n}\right) \leq|F|\left(\varepsilon_{n}\left(u_{0} \otimes x_{0}\right)\right)=\varepsilon_{n}|F|\left(u_{0} \otimes x_{n}\right)
$$


so $F\left(f_{n}\right) \rightarrow 0$, as desired. On the other hand, since $\frac{1}{n} y_{0}^{*} \stackrel{(w)}{\longrightarrow} 0$ in $Y^{*}$ and $T$ is supposed to be a weak Dunford-Pettis operator, we get $\frac{1}{n} y_{0}^{*}\left(T\left(f_{k_{n}}\right)\right) \rightarrow 0$, which contradicts $(*)$.

(ii) Assume that $T: E(X) \rightarrow Y$ is order-bounded. Then by Theorem $2.3 T$ is $(\sigma(E(X)$, $\left.\left.E(X)^{\sim}\right), \sigma\left(Y, Y^{*}\right)\right)$-continuous. Let $f_{n} \rightarrow 0$ for $\sigma\left(E(X), E(X)^{\sim}\right)$ and $y_{n}^{*} \stackrel{(w)}{\longrightarrow} 0$ in $Y^{*}$. Then $T\left(f_{n}\right) \stackrel{(w)}{\longrightarrow} 0$ in $Y$, and since $Y$ is supposed to have the Dunford-Pettis property, we conclude that $y_{n}^{*}\left(T\left(f_{n}\right)\right) \rightarrow 0$. This means that $T$ is a weak Dunford-Pettis operator.

\section{References}

[AB 1 C. D. Aliprantis and O. Burkinshaw, Locally Solid Riesz Spaces, Academic Press, New York, 1978.

$\left[\mathrm{AB}_{2}\right] \quad$ C. D. Aliprantis and O. Burkinshaw, Dunford-Pettis operators on Banach lattices, Trans. Amer. Math. Soc. 274 (1982), 227-238.

$\left[\mathrm{AB}_{3}\right]$ C. D. Aliprantis and O. Burkinshaw, Positive Operators, Academic Press, Orlando, 1985.

[B] A. V. Bukhvalov, On an analytic representation of operators with abstract norm, Izv. Vyss. Ucheb. Zaved. 11 (1975), 21-32 (in Russian).

[BL] A. V. Bukhvalov and G. Ya. Lozanovskii, On sets closed in measure in spaces of measurable functions, Trans. Moscow Math. Soc. 2 (1978), 127-148.

[D] P. G. Dodds, o-weakly compact mappings of Riesz spaces, Trans. Amer. Math. Soc. 214 (1975), 389-402.

[Du] M. Duhoux, o-weakly compact mappings from a Riesz space to a locally convex space, Bull. Math. Soc. Sci. Math. R.S. Roumanie 22 (70) (1978), 371-378.

[E] Z. Ercan, Interval-bounded operators and order-weakly compact operators of Riesz spaces, Demonstr. Math. 31 (1998), 805-812.

[F] D. Fremlin, Topological Riesz Spaces and Measure Theory, Camb. Univ. Press, 1974.

[KA] L. V. Kantorovich and A. V. Akilov, Functional Analysis, Nauka, Moscow 1984 (3 $3^{\text {rd }}$ ed., in Russian).

[Na] I. Namioka, Partially ordered linear topological spaces, Mem. Amer. Math. Soc. 24 (1957).

[N $\mathrm{N}_{1}$ M. Nowak, Duality theory of vector valued function spaces I, Comment. Math. 37 (1997), 195-215.

$\left[\mathrm{N}_{2}\right] \quad$ M. Nowak, Duality theory of vector-valued function spaces II, Comment. Math. 37 (1997), 217-230.

[W] A. Wilansky, Modern Methods in Topological Vector-Spaces, McGraw-Hill, 1978. 